\title{
Merely possible explanation
}

\author{
GHISLAIN GUIGON \\ eidos - The Genevan Centre for Metaphysics, Department of Philosophy, \\ University of Geneva, 1211 Geneva 4, Switzerland \\ e-mail: Ghislain.Guigon@unige.ch
}

\begin{abstract}
Graham Oppy has argued that possible explanation entails explanation in order to object to Richard Gale and Alexander Pruss's new cosmological argument that it does not improve upon familiar cosmological arguments. Gale and Pruss, as well as Pruss individually, have granted Oppy's inference from possible explanation to explanation and argue that this inference provides a reason to believe that the strong principle of sufficient reason is true. In this article, I shall undermine Oppy's objection to the new cosmological argument by arguing that it is logically possible that some truths are merely possibly explained.
\end{abstract}

Graham Oppy ${ }^{1}$ objects to Richard Gale and Alexander Pruss's new cosmological argument ${ }^{2}$ that it does not improve upon traditional cosmological arguments because the weak principle of sufficient reason invoked in the former entails the strong principle of sufficient reason invoked in the latter.

Let the strong principle of sufficient reason be the following: ${ }^{3}$

S-PSR For every proposition $p$, if $p$ is true then there is an explanation for $p$.

The weaker principle of sufficient reason Gale and Pruss appeal to is the following:

W-PSR For every proposition $p$, if $p$ is true then it is possible that there is an explanation for $p$.

Richard Gale and Alexander Pruss, as well as Pruss individually, ${ }^{4}$ grant the inference from the W-PSR to the S-PSR and claim that this inference provides a further reason to believe that the S-PSR is true.

In this article I shall argue that the W-PSR does not entail the S-PSR. I shall first argue that an ancillary assumption for the derivation from the W-PSR to the S-PSR, namely the assumption that explanation is dissective, is affirmed in the 
absence of good grounds. Then I shall argue that there is a good reason to deny that explanation is dissective. If it is sound, my reasoning shows that it is logically possible for some truths to be merely possibly explained and undermines one of the main objections against the new cosmological argument.

\section{Oppy's inference}

Gale and Pruss's new cosmological argument relies on the following definitions and assumptions:

Definition 1: A possible world is a maximal compossible conjunction of abstract propositions.

Definition 2: $\quad$ The big conjunctive fact (BCF) for a possible world is the conjunction of all the propositions that would be true if the world were actual.

Definition 3 The big conjunctive contingent fact (BCCF) for a possible world is the conjunction of all of the contingent propositions that would be true if the world were actual.

Assumption $1 \quad$ For any proposition $p$ and any world $w$, the BCF for $w$ either contains $p$, or the negation of $p$, but not both.

Assumption 2 For any proposition $p$ and any world $w$, if $p$ is in $w$ 's $\mathrm{BCF}$, then there is some world $w^{*}$ and proposition $q$ such that $p$ and $q$ are in $w^{* \prime}$ BCF, and $q$ explains $p$ (the W-PSR given definition 1 of a world).

Then the argument runs as follows:

(1) If $p_{1}$ is the BCCF for a world $w_{1}$, and $p_{2}$ is the BCCF for a world $w_{2}$, and $p_{1}=p_{2}$, then $w_{1}=w_{2}$.

[by definition 1 and definition 3]

(2) @ is the BCCF of the actual world.

[definition]

(3) For any proposition $p$ and any world $w$, if $p$ is in $w$ 's BCF, then there is some world $w^{*}$ and proposition $q$ such that $p$ and $q$ are in $w^{*}$ 's BCF, and $q$ explains $p$.

[the W-PSR]

(4) (Hence) there is a possible world $w^{\prime}$ and a proposition \# such that the BCF for $w^{\prime}$ contains @ and \#, and the proposition that \# explains @.

[from 2 and 3]

(5) $w^{\prime}=$ the actual world.

[from 1, definition 1, definition 2, and assumption 1]

(6) (Hence) in the actual world, there is a proposition \# such that the BCF for the actual world contains @, \#, and the proposition that \# explains@. 
Gale and Pruss argue further that \# has to be a contingent proposition that reports the free intentional action of a necessary being.

Oppy objects to the new cosmological argument is that the W-PSR entails the S-PSR and that since we have good reasons to reject the S-PSR, we have good reasons to reject the W-PSR. ${ }^{5}$ Oppy's derivation from the W-PSR to the S-PSR parallels the Fitch derivation, which is the derivation from the verificationist premise:

K1 For every $p$, if $p$ is true then it is possible to know that $p$.

to

K2 For every $p$, if $p$ is true then someone knows that $p$.

Briefly, the Fitch derivation runs as follows. Suppose that $\mathrm{K}_{1}$ is true and $\mathrm{K} 2$ is false. By the negation of $\mathrm{K} 2$ there is some proposition $q$ such that $q$ is true and no one knows that $q$. Substitute the proposition ( $q$ and no one knows that $q$ ) for $p$ in K1. Then, by modus ponens, we can infer that it is possible that someone knows that ( $q$ and no-one knows that $q$ ). Yet the latter is absurd provided we accept that knowledge is dissective:

DK For any propositions $p$ and $q$, if someone knows that $p \& q$, then someone knows that $p$ and someone knows that $q$.

For by DK we get the absurd result that it is possible that (some $x$ knows that $q$ and there is no $x$ who knows that $q$ ). Therefore, on the assumption that DK is valid, if $\mathrm{K}_{1}$ is true so is $\mathrm{K} 2$.

Which reasons are there to believe that $\mathrm{DK}$ is true on the assumption that $\mathrm{K} 2$ is false? The answer is trivial: it is impossible for a given knower to know a conjunction and fail to know each of its conjuncts. Thus, DK is motivated by the following stronger claim:

SDK For any propositions $p$ and $q$ and for any $x$, if $x$ knows that $p \& q$, then $x$ knows that $p$ and $x$ knows that $q$.

Oppy's derivation from the W-PSR to the S-PSR is a reductio that is complete on the presence of the following ancillary assumption about explanation that parallels DK:

DE For any propositions $p$ and $q$, if there is an explanation for $p \& q$, then there is an explanation for $p$ and there is an explanation for $q .^{6}$

Thus, that explanation is dissective.

Oppy's reductio runs as follows. Suppose that the W-PSR is true and that the S-PSR is false. If the S-PSR is false, then there is a proposition $q$ such that $q$ is true and there is no explanation for $q$. Substitute ' $q$ and there is no explanation 
for $q$ ' for $p$ in the W-PSR. Then, by modus ponens, we can infer that it is possible that there is an explanation for ( $q$ and there is no explanation for $q$ ). By DE we can derive that it is possible that (there is an explanation for $q$ and there is an explanation for there being no explanation for $q$ ). By the further principle that explanation is veridical-i.e. the undeniable claim that propositions having a true explanation are themselves true - we can infer that it is possible that (there is an explanation for $q$ and there is no explanation for $q$ ), which is absurd.

Oppy claims that DE is plausible. ${ }^{7}$ But which reasons are there to believe that $\mathrm{DE}$ is true? DE is trivially true on the assumption that the S-PSR is true. But since in the derivation from the W-PSR to the S-PSR we are assuming that the S-PSR is false, the S-PSR cannot be the motivation for DE here.

Some may suggest that the justification for DE is the claim that explanation is closed under entailment, i.e. the claim that, necessarily, for any $p, q$, and $r$, if $q$ is an explanation for $p$ and $p$ entails $r$, then $q$ is an explanation for $r$. But someone who, like Oppy, rejects the S-PSR would be well advised to deny that explanation is closed under entailment. For suppose that $p$ is a true proposition such that $q$ explains $p$, and suppose that $r$ is a logical necessity that has no explanation. Since $r$ is a logical necessity, $p$ entails $p \& r$. Then, if explanation is closed under entailment, $q$ explains $p \& r$. And if DE, which follows from the assumption that explanation is closed under entailment, is true, it follows that $q$ explains $r$. Contradiction!

In order to avoid the contradiction, we must either maintain that no proposition has an explanation or maintain that every logical necessity has an explanation. But scientific nihilism, i.e. the claim that no truth has an explanation, is highly implausible. And, although both claims are not inconsistent, the conjunction of the claim that every logical necessity has an explanation and of the negation of the S-PSR is unappealing. For, since they are true no matter what, logical necessities are among the best candidates for being unexplained truths. So someone who, like Oppy, rejects the S-PSR should better reject the claim that explanation is closed under entailment.

Therefore, on the assumption that the S-PSR is false and given that Oppy's derivation is inspired by the Fitch derivation, the most plausible motivation for $\mathrm{DE}$ is the following parallel to SDK:

SDE For any propositions $p, q$, and $r$, if $r$ explains that $p \& q$, then $r$ explains that $p$ and $r$ explains that $q$.

However, I shall argue that SDE - contrary to SDK - plausibly fails. If I am right and if it is true that SDE is the most plausible motivation for DE in the absence of the S-PSR, then DE is ill motivated and there is no good reason to believe that the S-PSR follows from the W-PSR. ${ }^{8}$ 


\section{Explanations for conjunctive truths}

First, what do we mean by 'explanation' in the context of a cosmological argument? Gale and Pruss contend that they can conceive of only two sorts of explanation: personal and scientific; where a personal explanation is a proposition that reports the intentional action of an agent, and a scientific explanation is a conjunction of law-like propositions, be they deterministic or only statistical, and of a proposition that reports the occurrence of some event. ${ }^{9}$

Since the view that causation occurs in virtue of the instantiation of dispositional properties seems to me prima facie plausible, I will also take into account the possibility of an explanation that is a conjunction of law-like propositions, be they deterministic or only statistical, and of a proposition that reports the instantiation of a dispositional property by some entity. However, in order to simplify the exposition I will omit reference to law-like propositions in what follows and assume that these constitute the background conditions for any explanation that is not personal. Therefore, I will assume that an explanation is either a proposition that reports the intentional action of an agent, or a proposition that reports the occurrence of an event (given some law-like conditions), or a proposition that reports the instantiation of some dispositional property by some entity (given some law-like conditions).

Now the relevant question when evaluating SDE is: what explains conjunctions of contingent truths? I shall consider four plausible alternatives:

(i) For any event or action $\mathrm{C}$, if the occurrence of $\mathrm{C}$ changes a state of the world in which $p \& q$ is false into a state of the world in which $p \& q$ is true, then the proposition that reports the occurrence of $\mathrm{C}$ explains that $p \& q$.

(ii) For any disposition $\mathrm{C}$ and entity $e$, if the manifestation of $\mathrm{C}$ by $e$ changes a state of the world in which $p \& q$ is false into one in which $p$ $\& q$ is true, then the proposition that reports that $e$ instantiates $\mathrm{C}$ explains that $p \& q$.

(iii) For any events or actions $\mathrm{C}$ and $\mathrm{D}$, if (a) the occurrence of $\mathrm{C}$ changes a state of the world in which $p$ is false into a state of the world in which $p$ is true and (b) the occurrence of $\mathrm{D}$ changes a state of the world in which $q$ is false into one in which $q$ is true, then the conjunction of the propositions that report the occurrence of $\mathrm{C}$ and the occurrence of $\mathrm{D}$ explains that $p \& q$.

(iv) For any dispositions $\mathrm{C}$ and $\mathrm{D}$ and entities $e$ and $f$, if ( $a$ ) the manifestation of $\mathrm{C}$ by $e$ changes a state of the world in which $p$ is false into a state of the world in which $p$ is true and $(b)$ the manifestation of $\mathrm{D}$ by $f$ changes a state of the world in which $q$ is false into one in which $q$ is true, then the conjunction of the propositions that report that $e$ instantiates $\mathrm{C}$ and that $f$ instantiates $\mathrm{D}$ explains that $p \& q$. 
Each of (i)-(iv) is a plausible claim about the explanation of conjunctions of contingent truths. Importantly, neither of (i) and (ii) implies that each of $p$ and $q$ has an explanation, whereas each of (iii) and (iv) implies that each of $p$ and $q$ has an explanation. So plausibly any failure of DE, if there is any, is a situation in which either (i) or (ii) holds. In the last section of this article, I exhibit such a plausible failure of DE.

However, I shall first argue that if either (i) or (ii) is the case, SDE is clearly false. Then I shall argue that if either (iii) or (iv) is the case, we should reject SDE.

\section{Plausible failures of SDE}

Suppose that I am giving a talk at a conference, that there is a bottle on the table in front of me, placed there by the organizer, and that I have a glass in my hand. Then, by placing the glass on the table I change a state of the world in which it is false that there is a bottle and a glass on the table into a state of the world in which it is true that there is a bottle and a glass on the table. Therefore, if (i) is the case, the proposition that I placed a glass on the table (together with some law-like propositions) explains that there is a bottle and a glass on the table. Yet it cannot be true that the proposition that I placed a glass on the table explains that there is a bottle on the table since the latter was true before I acted. Therefore, if (i) is the case, SDE is clearly false.

A similar reasoning shows that SDE fails if (ii) is the case. Suppose that the glass in front of me is broken and I have some opium in my hand. By taking the dose of opium I have in my hand I drift into sleep. In other words, my drifting into sleep is a manifestation of the soporific disposition of the dose of opium I had in my hand. My drifting into sleep changes a state of the world in which it is false that the glass is broken and I am asleep into a state of the world in which it is true that the glass is broken and I am asleep. By (ii), the proposition that the dose of opium I had in my hand is soporific (together with some law-like propositions) explains that the glass is broken and I am asleep. By SDE the proposition that the dose of opium I had in my hand is soporific explains that the glass is broken. Yet it cannot be true that the proposition that the dose of opium I had in my hand is soporific explains that the glass is broken since the latter was true before the dose of opium I had in my hand manifested its soporific disposition. Therefore, if (ii) is the case, SDE clearly fails.

Now I wish to argue that if (iii) is true, SDE should be rejected. In order to do so, I shall first argue that if (iii) is the case, SDE fails according to a popular theory of causation, namely the counterfactual theory of causation. Following David Lewis, many contemporary philosophers account for causation in terms of counterfactual dependence. Roughly, if $\mathrm{C}$ is a cause of $\mathrm{E}$, then if $\mathrm{C}$ were not to occur $\mathrm{E}$ would not occur. And so, if $q$ is the causal explanation for $p$, then if $q$ were not 
true - if the event or action reported by $q$ were not to occur $-p$ would not be true. I shall argue that, given (iii), SDE conflicts with the counterfactual analysis of causation.

Suppose that the organizer of the conference placed a bottle on the table and I, the speaker, placed a glass (and no bottle) on the table. Then, according to (iii), the proposition that the organizer placed a bottle on the table and I placed a glass on the table explains that there is a bottle and a glass on the table. By SDE, the proposition that the organizer placed a bottle on the table and I placed a glass on the table explains that there is a bottle on the table. However, the proposition that there is a bottle on the table does not counterfactually depend on the proposition that the organizer placed a bottle on the table and I placed a glass on the table. For suppose that I placed no glass on the table though the organizer placed a bottle on the table. Then, it is true that there is a bottle on the table but false that the organizer placed a bottle on the table and I placed a glass on the table. So, given (iii), SDE conflicts with the counterfactual analysis of causation.

Some might object that the displayed situation is a counter-example to the bare bones counterfactual analysis of causation instead of a counter-example to SDE. For it is well known that there are situations in which the effect does not counterfactually depend on its cause; these are cases of so-called causal pre-emption. ${ }^{10}$ Consider the following example of late pre-emption:

Assassin and Badgirl both put poison in Victim's coffee, which he drinks at noon. Assassin uses a fast-acting poison that kills within one hour. Badgirl uses a slow-acting poison that takes from one to two hours to kill. At 1.00 pm, Victim is dead. If only Badgirl had administered the poison, Victim would have been alive at $1.00 \mathrm{pm}$, but dead by 2.00 pm. ${ }^{11}$

Intuitively, Assassin's action is a cause of Victim's death while Badgirl's action is not. If so, the proposition that Assassin puts poison in Victim's coffee explains that Victim is dead at $2.00 \mathrm{pm}$. However, the proposition that Victim is dead at $2.00 \mathrm{pm}$ counterfactually depends neither on the proposition that Assassin puts poison in Victim's coffee nor on the proposition that Badgirl puts poison in Victim's coffee.

But there is a significant difference between cases of pre-emption and the situation with the bottle and the glass. That Assassin puts poison in Victim's coffee causally grounds that Victim is dead at $2.00 \mathrm{pm}$. In other words, Victim is dead at $2.00 \mathrm{pm}$ because of, in virtue of, Assassin's action. However, it cannot be true that there is a bottle on the table because the organizer placed a bottle on the table and I placed a glass on the table, or so I shall argue. Then, since a causal explanation is intended as describing what causally grounds the truth of the proposition that reports the effect, it cannot be true that the proposition that the 
organizer placed a bottle on the table and I placed a glass on the table explains that there is a bottle on the table.

People working on the logic of grounding usually endorse the following claims as axiomatic or derived (where 'the $p s$ ', 'the $q \mathrm{~s}^{\text {', }}$ and 'the $r \mathrm{~s}$ ' are plural variables denoting either one or more propositions) $:^{12}$

Grounding for conjunction For every $p$ and $q, p$ and $q$ together ground that $p \& q$ (where it is assumed that some propositions together ground a further one if and only if each of the former helps grounding the latter). Transitivity For every $p s, q s$, and $r s$, if the $p s$ ground the $q s$ and the $q s$ ground the $r s$, then the $p s$ ground the $r s$.

Grounding for conjunction is motivated by the claim that a conjunction is true in virtue of the truth of its conjuncts. Transitivity is motivated by the claim that if, in order to be true, some proposition(s) require(s) the truth of some proposition(s) which itself/themselves require(s) some further proposition(s) to be true in order to be true, then the former proposition(s) require(s) the latter proposition(s) to be true in order to be true.

By grounding for conjunction, the proposition that the organizer placed a bottle on the table and the proposition that I placed a glass on the table (together) ground that the organizer placed a bottle on the table and I placed a glass on the table. Let us assume for the sake of the argument that (iii) and SDE are both true in such a way that that the organizer placed a bottle on the table and I placed a glass on the table (causally) grounds that there is a bottle on the table. By transitivity, the proposition that the organizer placed a bottle on the table and the proposition that I placed a glass on the table (together) ground that there is a bottle on the table. However, there is no way the proposition that I placed a glass on the table can have helped grounding that there is a bottle on the table since the bottle was there before I acted. Hence, we should deny that the proposition that the organizer placed a bottle on the table and I placed a glass on the table explains that there is a bottle on the table. If (iii) is true, SDE should be rejected.

Finally, I shall argue that if (iv) is the case, SDE should be denied. Suppose that the manifestation of the fragility of the glass changes a state of the world in which it is false that the glass in front of me is broken into one in which it is true that it is broken, and suppose that the manifestation of the soporific disposition of the opium I had in my hand changes a state of the world in which it is false that I am asleep into one in which it is true that I am asleep. By (iv) the proposition that the glass is fragile and the opium I had in my hand is soporific explains that the glass is broken and I am asleep. By SDE the proposition that the glass is fragile and the opium I had in my hand is soporific explains that the glass is broken.

But even in a dispositionalist context, a causal explanation is intended as describing what causally grounds the truth of the proposition that reports the effect. 
Since, by grounding for conjunction, the proposition that the glass is fragile and the proposition that the opium I had in my hand is soporific (together) ground that the glass is fragile and the opium I had in my hand is soporific, then by transitivity, (iv), and SDE the proposition that the glass is fragile and the proposition that the opium I had in my hand is soporific (together) ground that the glass is broken. However, there is no way that the soporific disposition of the opium I had in my hand can have helped grounding that the glass is broken since the glass was broken before the dose of opium manifested its disposition.

\section{Plausible failures of DE}

So far I have argued that if either (i) or (ii) is true, then SDE clearly fails, and I have argued that if either (iii) or (iv) is the case, SDE should be rejected. Since each of (i)-(iv) is a plausible candidate account of the explanation of conjunctions of contingent truths, we must conclude that SDE plausibly fails. Then since SDE is the most plausible motivation for DE and since SDE plausibly fails, we must conclude that there is no good reason to believe that DE is true. But are there good reasons to believe that DE is false on the assumption that the S-PSR is false? I think so.

It seems to me that among the reasons why people tend to believe that $\mathrm{DE}$ is true is the false belief that any explanation for a conjunction is somehow incomplete if one of its conjuncts remains unexplained. It is the belief that for any propositions $p$ and $q$ if there is an explanation for $p$ but no explanation for $q$, then something must be added to the explanation for $p$ in order to get an explanation for $p \& q$. This belief is justified if $q$ is such that its truth requires an explanation, but it is misguided if $q$ is a truth that requires no explanation.

As I emphasized when discussing whether explanation is closed under entailment, logical necessities are among the best candidates for being unexplained truths since they are true no matter what. Logical necessities do not only seem to be true in the absence of any explanation, they also seem to be such that their truth requires no explanation (where for any $p, p$ requires an explanation for its truth if, and only if, there is a set $x$ of propositions such that, for any world $w$, if $p$ is true in $w$, then some member of $x$ is true in $w$ and explains $p$ in $w$ ). For instance, the proposition that if $2+2=5$, then $2+2=5$ seems true independently of any explanation.

Now suppose that the proposition that there is a glass on the table requires an explanation in order to be true, and that its actual explanation is the proposition that I placed a glass on the table. Then, the proposition that I placed a glass on the table contributes to the explanation of the proposition that there is a glass on the table, and if $2+2=5$, then $2+2=5$. For my placing a glass on the table changes a state of the world in which it is false that there is a glass on the table and if $2+2=5$ 
then $2+2=5$, into a state of the world in which it is true that there is a glass on the table and if $2+2=5$, then $2+2=5$.

Moreover, assuming that the proposition that if $2+2=5$, then $2+2=5$ requires no explanation for its truth, the proposition that I placed a glass on the table clearly suffices to explain that there is a glass on the table and if $2+2=5$, then $2+2=5$. In other words, once I have explained why there is a glass on the table, nothing needs to be added to explain why there is a glass on the table and if $2+2=5$, then $2+2=5$.

Finally, since the proposition that I placed a glass on the table contributes to the explanation of the proposition that there is a glass on the table and if $2+2=5$, then $2+2=5$, and since its contribution to the explanation of this conjunction suffices to explain it, we must conclude that the proposition that I placed a glass on the table fully explains that there is a glass on the table and if $2+2=5$, then $2+2=5$. If so, the proposition that there is a glass on the table and if $2+2=5$, then $2+2=5$ has an explanation, even if one of its conjuncts has no explanation. ${ }^{13}$ Therefore, we are justified in rejecting DE on the grounds that some proposition requires no explanation for its truth.

Let me emphasize that nothing essential in the latter course of reasoning relies on my appeal to a logical necessity. What matters here is that one of the conjuncts requires no explanation in order to be true. On the assumption that the S-PSR is false, there is a proposition that is true and has no explanation. Then, since it follows from the above-mentioned definition of a proposition that requires an explanation for its truth, that every truth that lacks an explanation is a proposition that requires no explanation for its truth, substituting any proposition that lacks an explanation for the proposition that if $2+2=5$, then $2+2=5$ in the previous course of reasoning yields the same conclusion: namely, that we are justified in rejecting DE.

Importantly, the affirmation that truths that lack an explanation are propositions that require no explanation for their truth helps to make more plausible the central thesis of this article, that is, the thesis that it is logically possible that some truths are merely possibly explained. For if the W-PSR is true, the S-PSR is false, and DE is false, then there is an unexplained truth $q$ such that in some possible world $w$ there is an explanation for ( $q$ and there is no explanation for $q$ ) without there being an explanation for $q$. Yet, as an anonymous referee for Religious Studies rightly pointed out to me, we might legitimately wonder what an explanation for ( $q$ and there is no explanation for $q$ ) could be if it does not explain $q$. As s/he suggested to me, the most plausible explanation for ( $q$ and there is no explanation for $q$ ) is an explanation of why there is no explanation for $q$; yet it is unclear how an explanation for there being no explanation for $q$ could explain that ( $q$ and there is no explanation for $q$ ). However, we gain clarity once we consider that $q$ must be a proposition that requires no explanation for its truth since $q$ is true in the actual world but has no explanation in 
the actual world. It is because $q$ requires no explanation for its truth that explaining why there is no explanation for $q$ suffices to explain the whole conjunction. ${ }^{14}$

Since DE is affirmed in the absence of compelling grounds and since there is a good reason to deny DE on the assumption that the S-PSR is false, the reductio from the W-PSR to the S-PSR is not complete and the claim that the W-PSR entails the S-PSR is unjustified and plausibly false. If so, and since Oppy's main objection against the new cosmological argument is not conclusive, the new cosmological argument is significantly distinct from traditional arguments involving the S-PSR. ${ }^{15}$

\section{Notes}

1. Graham Oppy “On “A new cosmological argument”', Religious Studies, 36 (2000), 345-353. Oppy’s objection is reproduced in idem Arguing about Gods (Cambridge: Cambridge University Press, 2006), 131-132, and in idem Philosophical Perspectives on Infinity (Cambridge: Cambridge University Press, 2006), 281-282.

2. Richard M. Gale \& Alexander R. Pruss 'A new cosmological argument', Religious Studies, 35 (1999), 461-476.

3. I follow the convention of intending but suppressing a necessity operator in front of every claim labelled in capital letters.

4. Richard M. Gale \& Alexander R. Pruss 'A response to Oppy, and to Davey and Clifton', Religious Studies, 38 (2002), 89-99. See also Alexander R. Pruss The Principle of Sufficient Reason: A Reassessment (Cambridge: Cambridge University Press, 2006), 234-235.

5. Oppy 'On “A new cosmological argument”', 346-348.

6. Ibid., 347 .

7. Ibid.

8. As an anonymous referee for Religious Studies, to whom I am grateful, pointed out to me, the claim that SDE plausibly fails also seems to challenge Gale \& Pruss's argument in favour of the view that @'s explanation has to be a personal one; Gale \& Pruss 'A new cosmological argument', 465. Their argument runs as follows. Let '@' stand for the BCCF for the actual world and '\#' for the alleged explanation for @. We can conceive of only two sorts of explanation, personal and scientific. Suppose for reductio that \# is a scientific explanation. A scientific explanation contains some law-like proposition and a proposition that reports the occurrence of some event. Such a proposition is contingent, and so \# is contained in@. If so, since \# must explain each and every contingent conjunct of @ as well as the conjunction as a whole, \# has to explain itself. However, this is impossible because no scientific explanation can explain itself. Therefore by reductio, \# is a personal explanation. It can be objected to this argument that the only reason to accept that \# has to explain each and every conjunct of @ as well as @ as a whole is the belief that SDE is true. So if I am right that SDE plausibly fails, it appears that the new cosmological argument fails to establish that the explanation of the universe is not a scientific explanation, and thus does not establish that there is an orthodoxly conceived monotheistic God.

9. Gale \& Pruss 'A new cosmological argument', 465.

10. On cases of pre-emption, see e.g. David K. Lewis 'Causation as influence', in John Collins, Ned Hall, \& Laurie A. Paul (eds) Causation and Counterfactuals (Cambridge MA: MIT Press, 2004), 75-106; and Christopher Hitchcock 'Prevention, preemption, and the principle of sufficient reason', Philosophical Review, 116 (2007), 495-532.

11. Hitchcock 'Prevention, preemption, and the principle of sufficient reason', 524.

12. See Fabrice Correia 'Grounding and truth-functions', forthcoming in Logique et analyse; Kit Fine 'Guide to ground' (manuscript); and Benjamin Schnieder 'A logic for "because”, (manuscript).

13. Given our assumptions, its explanation is of type (i); see section 'Explanations for conjunctive truths'. 
14. It follows from the previous reasoning that, if $q$ is contingent, then, in worlds where $q$ is false, explaining why $q$ is not explained does not suffice to explain why $q$ is false and not explained. Here we would need a further explanation for the falsity of $q$, and this would be an explanation of why $\neg q$ is true. It also follows from the previous reasoning that some unexplained truth could have been explained although it requires no explanation for its truth. But this is exactly what we mean when saying that atheism is a contingent truth: we mean that the universe could have been explained even though it need not be explained since it is not explained.

15. I am indebted to the participants to the eidos metaphysics seminar 2009-2010, to Fabrice Correia, Graham Oppy, and to an anonymous referee for Religious Studies for their helpful comments on earlier versions of this paper. 